\title{
Influence of synovia constituents on tribological behaviors of articular cartilage
}

\author{
Teruo MURAKAMI ${ }^{1,}$, , Seido YARIMITSU ${ }^{\mathfrak{1}}$, Kazuhiro NAKASHIMA ${ }^{2}$, Yoshinori SAWAE, Nobuo SAKAI ${ }^{3}$ \\ ${ }^{1}$ Research Center for Advanced Biomechanics, Kyushu University, 744 Motooka, Nishi-ku, Fukuoka, 819-0395, Japan \\ ${ }^{2}$ Department of Mechanical Engineering, Faculty of Engineering, Kyushu University, 744 Motooka, Nishi-ku, Fukuoka, 819-0395, Japan \\ ${ }^{3}$ Department of Applied Science for Integrated System Engineering, Graduate School of Engineering, Kyushu Institute of Technology, \\ 1-1, Sensuicho, Tobata-ku, Kitakyushu, 804-8550, Japan
}

Received: 31 December 2012 / Revised: 18 February 2013 / Accepted: 16 March 2013

(C) The author(s) 2013. This article is published with open access at Springerlink.com

\begin{abstract}
The extremely low friction and minimal wear in natural synovial joints appear to be established by effective lubrication mechanisms based on appropriate combination of articular cartilage and synovial fluid. The complex structure of cartilage composed of collagen and proteoglycan with high water content contributes to high load-carrying capacity as biphasic materials and the various constituents of synovial fluid play important roles in various lubrication mechanisms. However, the detailed differences in functions of the intact and damaged cartilage tissues, and the interaction or synergistic action of synovia constituents with articular cartilage have not yet been clarified. In this study, to examine the roles of synovia constituents and the importance of cartilage surface conditions, the changes in friction were observed in the reciprocating tests of intact and damaged articular cartilage specimens against glass plate lubricated with lubricants containing phospholipid, protein and/or hyaluronic acid as main constituents in synovial fluid. The effectiveness of lubricant constituents and the influence of cartilage surface conditions on friction are discussed. In addition, the protectiveness by synovia constituents for intact articular cartilage surfaces is evaluated.
\end{abstract}

Keywords: articular cartilage; synovial fluid; synovial joint; lubrication; biotribology

\section{Introduction}

In various biotribological systems, it is widely known that the healthy synovial joints maintain superior load-carrying capacity and lubricating properties with extremely low friction and minimal wear even in heavily loaded hip, knee and ankle joints. The synovial joints are prominent natural bearings different in geometric congruity depending on joint positions/ movements and are in general covered with soft layers of biphasic articular cartilage lubricated with synovial fluid containing appropriate lubricating constituents. The superior tribological properties of synovial joints appear to be established by a well-suited combination

* Corresponding author: Teruo Murakami.

E-mail: tmura@mech.kyushu-u.ac.jp of articular cartilage and synovial fluid. However, the detailed cooperative and/or interactive behaviors between articular cartilage and synovial fluid under various rubbing conditions have not yet been clarified. In this paper, we will focus on the influence of main synovia constituents such as phospholipid, protein and hyaluronic acid on tribological behaviors of articular cartilage different in surface conditions particularly as related to lubrication mechanism.

The operating conditions in human synovial joints change under variable loading and motions including sliding and rolling depending on joint types in various daily activities. Therefore, the superior lubricating performance of natural synovial joints is likely to be actualized not by a single lubrication mode but by the synergistic combination of various modes from fluid film lubrication to boundary lubrication [1, 2]. 
Other specific lubrication mechanisms such as weeping lubrication [3], boosted lubrication [4], biphasic lubrication [5], micro-elastohydrodynamic lubrication (micro-EHL) [6] and so on have been proposed. The ingenious lubrication mechanism as the synergistic combination of various modes depending on the severity of operating conditions was called the adaptive multimode lubrication mechanism [7, 8]. For example, during normal walking, fluid film lubrication mechanisms such as soft-EHL and/or micro-EHL play major roles to maintain low friction and minimize wear. In contrast, in thin film conditions such as at slow motion or at movement after standing for a long time, it is expected that adsorbed films [9-12], surface gel films [13], hydration lubrication [14] and polymeric brush-like layers $[15,16]$ contribute to keep friction low and protect rubbing surfaces.

Another new development in lubrication theory is the elucidation of the biphasic lubrication mechanism. Since an experimental finding [17] and a proposal of boundary friction model based on biphasic lubrication by Ateshian [18], the important phenomena on the effectiveness of biphasic lubrication with interstitial fluid pressurization have been demonstrated on the basis of the biphasic finite element (FE) analyses and experimental observations [19, 20]. The articular cartilage has high water content from $70 \%$ to $80 \%$ in tissue as porous media composed of type II collagen, proteoglycan and chondrocytes, and thus exhibits a time-dependent biphasic behavior due to the simultaneous coexistence of solid and liquid phases [21]. When articular cartilage as biphasic material with low permeability is applied by compressive load, the fluid content in the tissue is trapped within contact area and the collagen matrix network resists interstitial fluid pressure in aggregate solid matrix. Thus, the interstitial fluid pressure supports significant proportion of total load in contact area and this situation consequently causes the reduction of contact force of solid phase for a considerable time. The timedependent change in load support by interstitial fluid pressure in biphasic cartilage depends on the extent of exudation from cartilage tissue and rehydration of cartilage. If the fluid load support is maintained at high level for a long time, the low friction is maintained because of low level for solid-to-solid contact [20].
For reciprocating sliding under constant load, Pawaskar et al. [22] introduced sliding motion into their FE model and indicated the importance of migrating contact area for the sustainability of the biphasic lubrication in their biphasic FE analysis. Sufficient stroke for rehydration of cartilage tissue in reciprocating motion maintained the high level of load support by interstitial fluid pressure. Sakai et al. [23] examined the compressive response of the articular cartilage by high precision testing machine with a feedback-controlled servomotor and estimated material properties in physiological condition for the biphasic FE model, which included (1) the depth-dependence of apparent Young's modulus of solid phase, (2) straindependent permeability as compaction effect, and (3) collagen reinforcement in tensile strain. These properties (parameters) were estimated by the curve fitting between the experimental time-dependent compressive behavior and simulation in indentation tests for cartilage specimens with cylindrical rigid indenter of $5 \mathrm{~mm}$ radius. In the reciprocating test, the load of $0.5 \mathrm{~N} / \mathrm{mm}$ was applied at the center of the cylindrical indenter in $1 \mathrm{~s}$ and then the reciprocating motion was introduced with the speed of $4 \mathrm{~mm} / \mathrm{s}$ over a stroke length of $8 \mathrm{~mm}$. FE analyses using commercial package ABAQUS (6.8-4) showed that the tensile reinforcement by spring elements representing the collagen network and the depth-dependent elastic properties improved the proportion of the fluid load support especially in the sliding condition. The compaction effect on permeability of solid phase was functional in a condition without the migrating contact area, whereas under sliding condition the compaction effect showed a little effect in terms of the proportion of the fluid load support.

In the next stage, the influence of operating conditions on the effectiveness of biphasic lubrication in reciprocating sliding was examined. The differences in frictional behaviors between the reciprocation with migration of contact zone, i.e., at on-off loading on articular cartilage (model A) as described above, and without migration of contact zone, i.e., at continuous loading on cartilage (model B), shown in Fig. 1 were compared in FE analysis [24]. In this simulation of reciprocating test with similar method to the previous study [23], the load of $0.5 \mathrm{~N} / \mathrm{mm}$ was applied by 


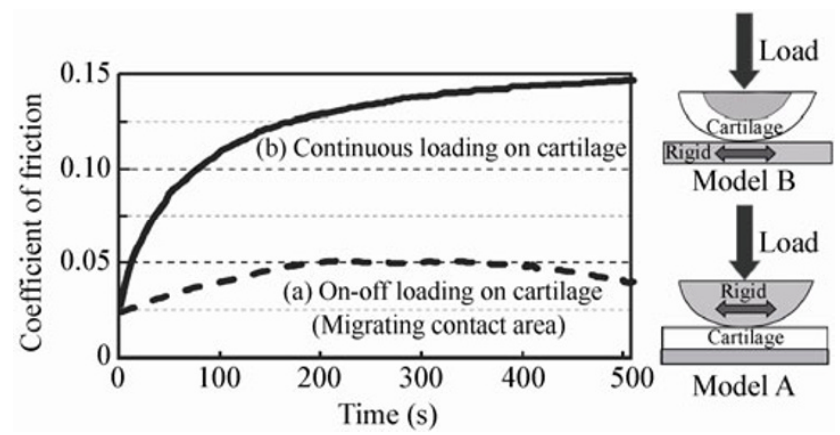

Fig. 1 Time-dependent frictional behaviors estimated by biphasic theory for cartilage.

the rigid cylindrical indenter against flat cartilage specimen or by the rigid flat plate against cylindrical cartilage specimen with a ramp time of $1 \mathrm{~s}$ and then the load was held constant during reciprocation. The reciprocation of rigid cylinder or flat plate at $4 \mathrm{~mm} / \mathrm{s}$ was started immediately after loading and continued for $508 \mathrm{~s}, 127$ cycles at period of $4 \mathrm{~s}$. The initial fluid load support percentages are very high as $90 \%$ and 91\% for models A and B, respectively. After 127 cycles, it is noticed that the high percentage of fluid load support (83\%) was maintained even after $508 \mathrm{~s}$ in model A, but the percentage of fluid load support was remarkably decreased to $27 \%$ in the model $\mathrm{B}$. The time-depending changes in friction coefficient $\mu_{\text {eff }}$ were estimated for $\mu_{\text {eq }}$ as coefficient of friction for solid-to-solid contact using the following formula by Ateshian et al. [20, 25].

$$
\mu_{\text {eff }}=\mu_{\mathrm{eq}}\left(1-(1-\Psi) W_{\mathrm{p}} / W\right)
$$

where $W$ is the total load support, $W_{\mathrm{p}}$ the load support by fluid pressure and $\Psi$ the fraction of contact area of solid phase.

In Fig. 1, the time-dependent changes in friction estimated from total traction force in biphasic FE analysis for assumption of $\mu_{\mathrm{eq}}=0.2$ [24] are shown. It is worth noting that the lower friction level is maintained due to the sustainability of interstitial fluid pressure in the reciprocating sliding for model A. In contrast, significant gradual increase to high level in friction is observed in reciprocation for model B. It is supposed that the tribological problems are more likely to occur for model B with high friction level and thus the method to suppress friction increase is required.
In this study, the combination of cartilage-on-glass was used to simplify the frictional condition, although articular cartilage is rubbed against cartilage or meniscus in natural synovial joints. The glass plate has very smooth, hard and non-porous/impermeable surface compared with articular cartilage but hydrophilic surface with negatively charged property similar to proteoglycan on superficial cartilage layer in wet condition [12]. The adsorption of synovia constituents on glass plate appears to be considerably similar to boundary film formation on intact cartilage as shown by in situ observation for fluorescent images of adsorbed molecules during reciprocating rubbing process [26], while the interaction to the smooth, hard and non-porous/impermeable glass surface may induce certain different behaviors. Smooth glass surface minimizes ploughing resistance, but may enhance the adhesive resistance by interaction with adsorbed protein molecules at intimate contacts in very thin film condition. However, the intrinsic tribological properties of compliant and biphasic articular cartilage are expected to be reflected appropriately in the effectiveness of lubricant constituents even in sliding pair of articular cartilage and glass plate. As a matter of course, the difference in tribological behaviors between for cartilage-cartilage and cartilage-glass combinations should be explored. The influence of glass plate on frictional behaviors is discussed in Section 4. Thus, the frictional behaviors in a sliding pair of ellipsoidal articular cartilage specimens and reciprocating glass plate were examined in the sliding condition for model B without migration of contact zone for cartilage.

\section{Materials and methods}

The reciprocating test for the sliding pair of the upper stationary ellipsoidal articular cartilage specimen and the lower reciprocating flat glass plate was conducted in the reciprocating tester shown in Fig. 2. The continuous loading condition without migration of contact zone for articular cartilage corresponds to the severe operating condition for cartilage (model B) as described above in related to the biphasic FE analysis. 


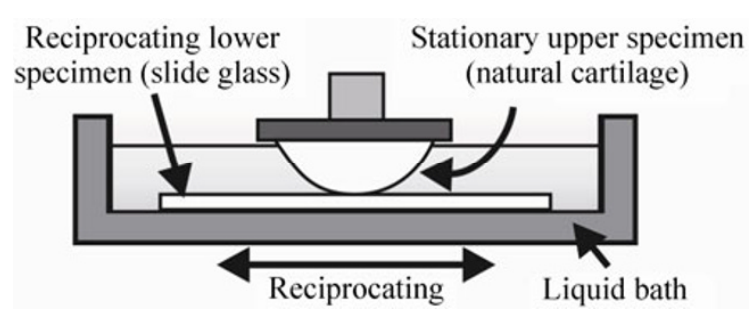

Fig. 2 Reciprocating apparatus.

\subsection{Materials}

An upper intact cartilage specimen with subchondral layer was prepared from a femoral condyle in a porcine knee joint ( 6 to 7 months old). The damaged cartilage specimen was prepared by wiping 15 times with a wiping tissue (Kimwipe), where the partial removal of surface gel layer was confirmed by observation with atomic force microscopy (AFM). AFM images in tapping mode (in Dimension Icon, Bruker Corpration, USA) in saline solution for intact and damaged specimens are shown in Fig. 3. On the damaged cartilage surface, the partial removal of surface gel-like layer is recognized with some exposed collagen fibers. The glass plate as a lower specimen is a slide glass.

The lubricants are saline solution containing $0.15 \mathrm{M}$ $\mathrm{NaCl}$ (Otsuka Pharmaceutical Factory Inc., Japan), saline solution of $0.5 \mathrm{wt} \%$ sodium hyaluronate (HA, molecular weight: $\left.9.2 \times 10^{5}\right)$, HA solutions containing $0.7 \mathrm{wt} \%$ or $1.4 \mathrm{wt} \%$ bovine serum albumin (Wako Pure Chemical Industries Ltd., Japan) and/or $0.7 \mathrm{wt} \%$ human serum $\gamma$-globulin (Wako Pure Chemical Industries Ltd., Japan) and/or $0.01 \mathrm{wt} \%$ L $\alpha$-dipalmitoyl phosphatidylcholine (L $\alpha$-DPPC) as an phospholipid liposome. In order to prevent bacterial growth in protein solutions as lubricant, $0.3 \mathrm{wt} \%$ sodium azide (a)

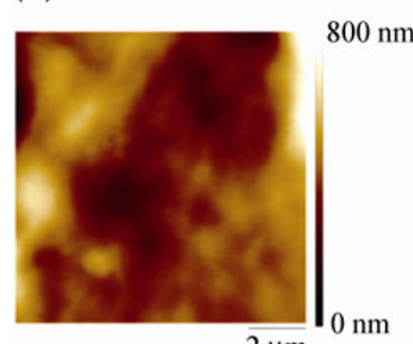

$2 \mu \mathrm{m}$ (b)

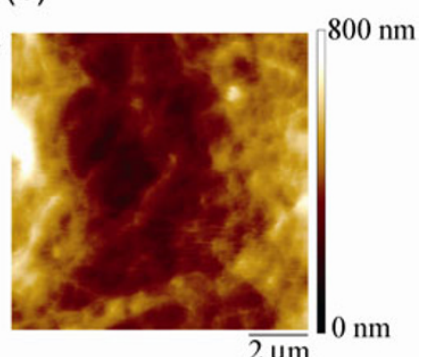

Fig. 3 AFM images of articular cartilage surfaces in saline solution: (a) intact cartilage and (b) damaged cartilage. was added for protein solutions. The combinations of lubricant constituents used in reciprocating tests are shown in Table 1.

Table 1 Compositions of lubricants (wt $\%$ ) as saline solutions.

\begin{tabular}{ccccc}
\hline & $\begin{array}{c}\text { HA:sodium } \\
\text { hyaluronate }\end{array}$ & DPPC & Albumin & $\gamma$-globulin \\
\hline 1 & 0 & 0 & 0 & 0 \\
2 & 0 & 0 & 0.7 & 0 \\
3 & 0 & 0 & 0 & 0.7 \\
4 & 0.5 & 0 & 0 & 0 \\
5 & 0.5 & 0 & 0.7 & 0 \\
6 & 0.5 & 0 & 0 & 0.7 \\
7 & 0 & 0.01 & 0 & 0 \\
8 & 0 & 0.01 & 0.7 & 0 \\
9 & 0 & 0.01 & 0 & 0.7 \\
10 & 0.5 & 0.01 & 0 & 0 \\
11 & 0.5 & 0.01 & 0.7 & 0 \\
12 & 0.5 & 0.01 & 0 & 0.7 \\
13 & 0.5 & 0.01 & 1.4 & 0.7 \\
\hline
\end{tabular}

\subsection{Experimental methods}

The reciprocating test was conducted at a sliding speed of $20 \mathrm{~mm} / \mathrm{s}$ for rectangular reciprocating mode and at a stroke of $35 \mathrm{~mm}$ at a constant load of $9.8 \mathrm{~N}$. The glass plate was cleaned ultrasonically in a solution of $0.5 \mathrm{vol} \%$ Triton X-100, distilled water and ethanol, and then dried. The lubricants were supplied in liquid bath. At room temperature, the reciprocating sliding was started immediately after loading, and interrupted after 514 cycles at sliding distance of $36 \mathrm{~m}$ for running time of $30 \mathrm{~min}$, and then the unloading state was maintained for $5 \mathrm{~min}$. Subsequently, the reciprocating test was restarted immediately after reloading and continued for a further sliding distance $36 \mathrm{~m}$. The restarting processes after unloading were repeated three times. The changes in friction force were continuously monitored to compare the differences in time-dependent frictional behaviors. The number of tests under the same condition was three.

\section{Results}

Time-dependent frictional behaviors for intact cartilage lubricated with saline, saline solutions of albumin, $\gamma$-globulin, HA and DPPC are shown in Fig. 4 . It is noted that the initial friction is very low between 0.01 


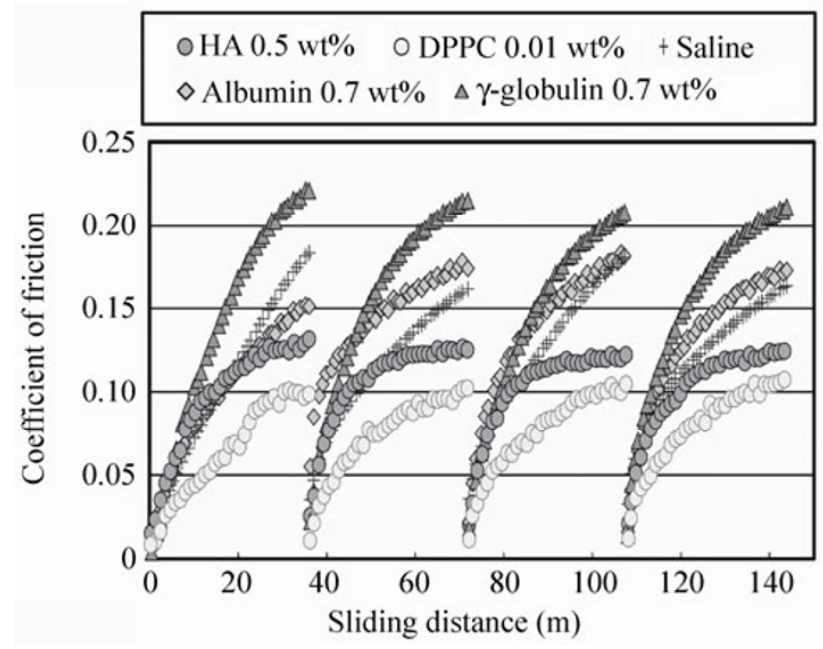

Fig. 4 Influence of lubricant constituents on frictional behaviors for intact articular cartilage.

and 0.02 as coefficient of friction for all lubricants, as typical for intact natural articular cartilage. However, the friction gradually increases with sliding distance until the sliding stops at $36 \mathrm{~m}$. The final values are different as the order of $\gamma$-globulin $>$ saline $>$ albumin $>$ HA $>$ DPPC. The addition of a single constituent into saline usually reduced the friction level at the final stage except $\gamma$-globulin. For $\gamma$-globulin, initial friction is lower than saline but the friction gradually increases to a higher level with thinning of lubricating film. At reloading-restarting after $5 \mathrm{~min}$ unloading at $36 \mathrm{~m}$ sliding, the restarting friction is remarkably reduced from the previous high level at interruption, but it is slightly higher than the initial friction, as reported by Murakami et al. [26, 27]. This friction reduction was considered to be brought by the recovery of both the hydration and some deformation of articular cartilage, in which the hydration lubrication and biphasic lubrication becomes partly effective accompanied with adsorbed film formation although initial adsorbed film may have been partly removed. In the second reciprocating sliding process, the friction again gradually increases with sliding distance. In the second to fourth processes where the cartilage surface was partly injured, albumin showed higher friction than the saline (Fig. 4).

The results mentioned above indicate the limitation of effectiveness of single additive for improvement of steady or final friction at each $36 \mathrm{~m}$ sliding. Therefore, it is required to examine the possibility in which the combination of different synovia constituents should be effective. As reported by our previous study [26], on the reduction of final friction at each $36 \mathrm{~m}$ sliding, the synergistic effect of $\gamma$-globulin and HA was confirmed, but the coexistence of albumin and HA showed the adverse interaction for intact and damaged cartilage. It was considered that the combination of HA and $\gamma$-globulin form adsorbed film cooperatively, and furthermore HA as a viscosity improver is likely to alleviate the friction resistance by its viscous property to improve the fluid film formation in a mixed lubrication regime. It was pointed out for the combination of albumin and HA that the repulsive properties of negatively charged molecules prevented the lubricating adsorbed film formation. In this study, the lubricity in the combination of DPPC and albumin or $\gamma$-globulin was examined for intact and damaged cartilage specimens. As shown in Fig. 5 for intact articular cartilage, the coexistence of DPPC and albumin or $\gamma$-globulin reduced friction compared with DPPC alone in saline. In contrast, some interaction between DPPC and proteins brought increase in friction for damaged roughened cartilage surfaces with partially removed gel-like layer as shown in Fig. 6 .

Next, the effectiveness of DPPC to HA solutions with and without proteins is evaluated. Figure 7 shows frictional behaviors for intact cartilage. It is noteworthy that even the addition of $0.01 \mathrm{wt} \%$ DPPC alone to HA solution exhibited a remarkable reduction in friction. Furthermore, the addition of $0.01 \mathrm{wt} \%$ DPPC accompanied with $1.4 \mathrm{wt} \%$ albumin, $0.7 \mathrm{wt} \%$-globulin

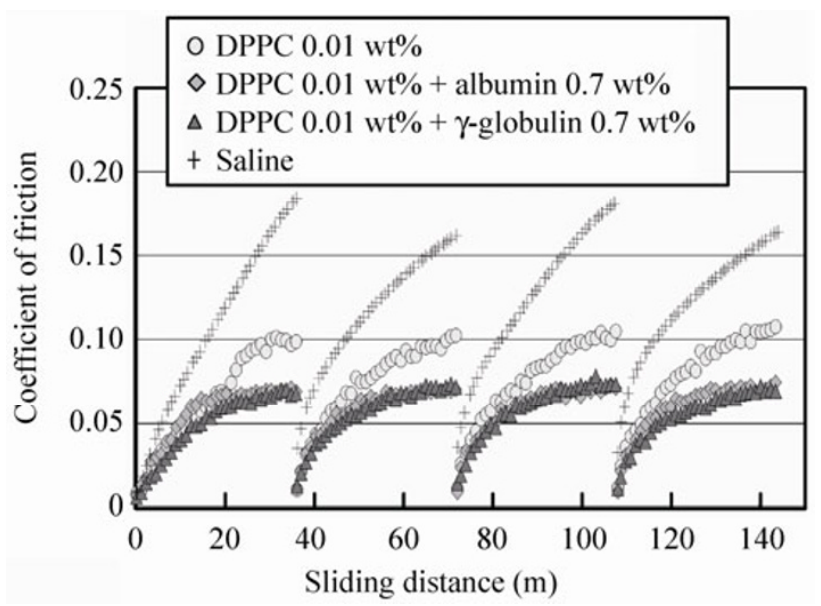

Fig. 5 Influence of DPPC and proteins on frictional behaviors for intact articular cartilage. 


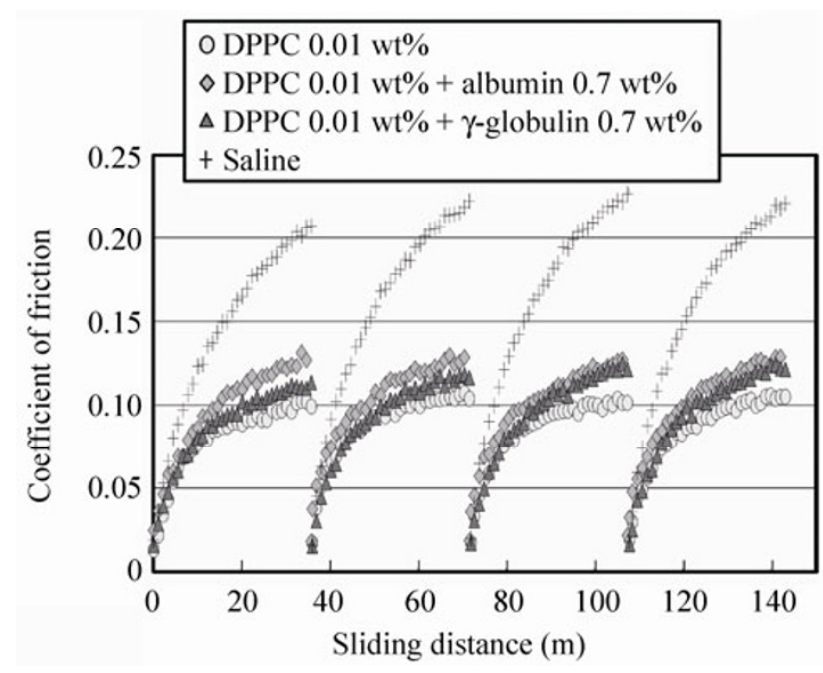

Fig. 6 Influence of DPPC and proteins on frictional behaviors for damaged articular cartilage.

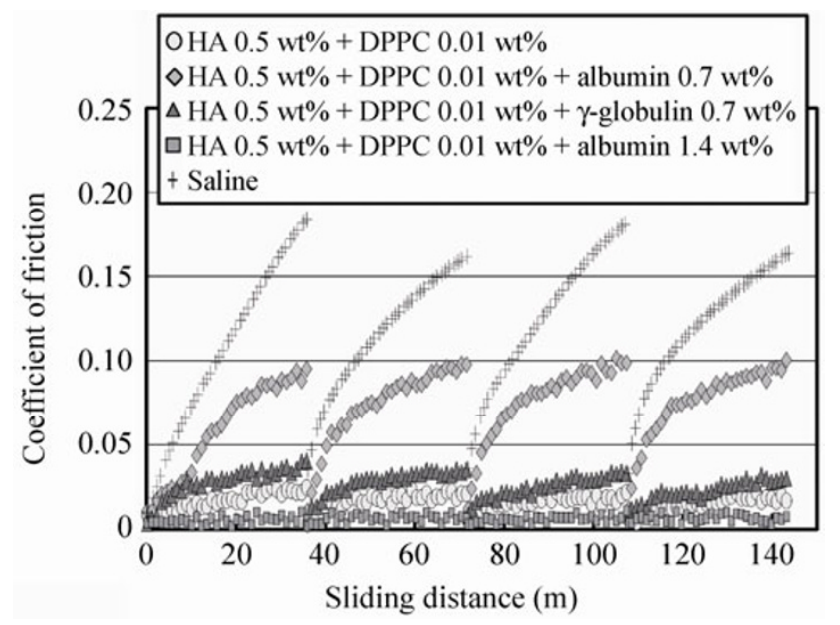

Fig. 7 Influence of DPPC, proteins and HA on frictional behaviors for intact articular cartilage.

in HA solution demonstrated the lowest frictional behaviors as about 0.01 without gradual increase until each $36 \mathrm{~m}$ sliding. In this lubricant composition, it is confirmed that the friction does not increase but maintains a very low steady level. On the contrary, the addition of DPPC with albumin alone or $\gamma$-globulin alone in HA solution shows higher friction than DPPC alone in HA solution but lower than saline solution. In Fig. 8 the frictional behaviors of these combinations for damaged cartilage are shown. Compared with intact cartilage, the friction levels are generally increased and the order of friction level is partly changed, i.e., HA solution containing DPPC

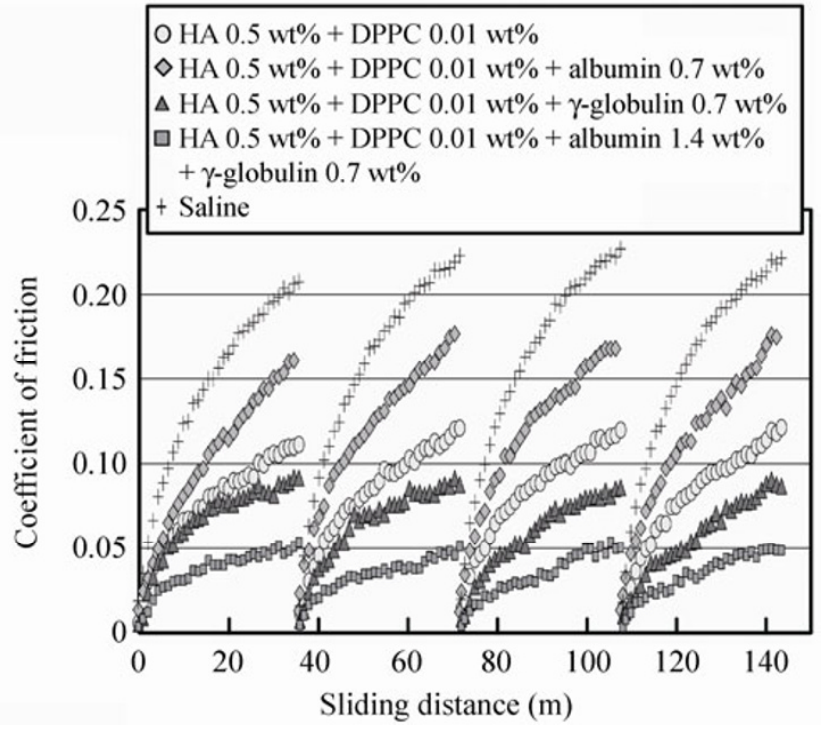

Fig. 8 Influence of DPPC, proteins and HA on frictional behaviors for damaged articular cartilage.

becomes higher than HA solution containing DPPC and $\gamma$-globulin. HA solution with $0.01 \mathrm{wt} \%$ DPPC, $1.4 \mathrm{wt} \%$ albumin and $0.7 \mathrm{wt} \% \gamma$-globulin maintains minimum friction but friction gradually increases until the level of 0.05 as coefficient of friction at each $36 \mathrm{~m}$ sliding for damaged cartilage.

The comparison of friction at restart and at steady state for both intact and damaged cartilage is summarized in Fig. 9. It is noteworthy for intact articular cartilage that the lubricant of HA solution with $0.01 \mathrm{wt} \%$ DPPC, $0.14 \mathrm{wt} \%$ albumin and $0.7 \mathrm{wt} \%$ $\gamma$-globulin showed the minimum coefficient of friction 0.003 and 0.01 at restart and at steady state, respectively. For damaged cartilage, these values showed 0.004 and 0.05 , respectively. It was confirmed that the optimum combination of DPPC, albumin and $\gamma$-globulin with HA for minimum friction is common (No. 13 in Table 1) for intact and damaged cartilage specimens.

To sustain superior tribological properties of articular cartilage, not only low friction but minimum wear are required in various daily activities. Therefore, wear on cartilage surfaces was evaluated. The articular cartilage contains plenty of water, therefore, it is difficult to measure actual changes due to wear in weight. In this study, the changes in surface photographs were compared with a surface before testing. Representative photographs are shown in Fig. 10. Intact 


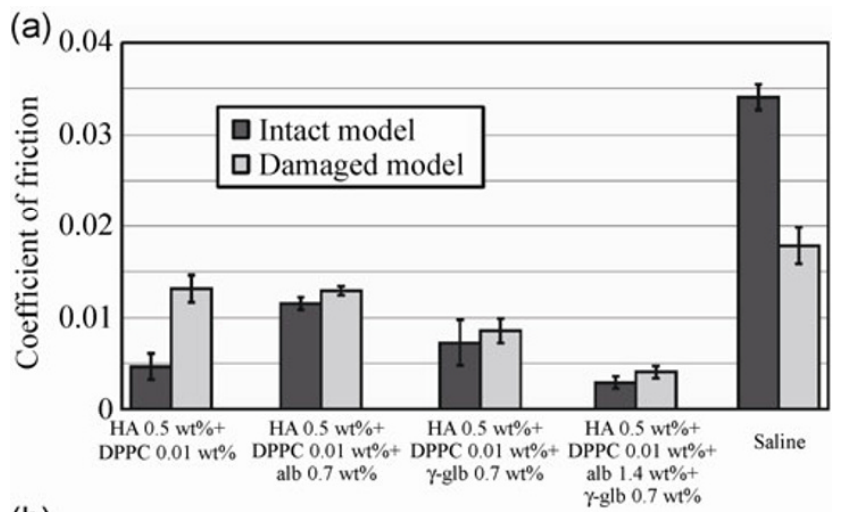

(b)

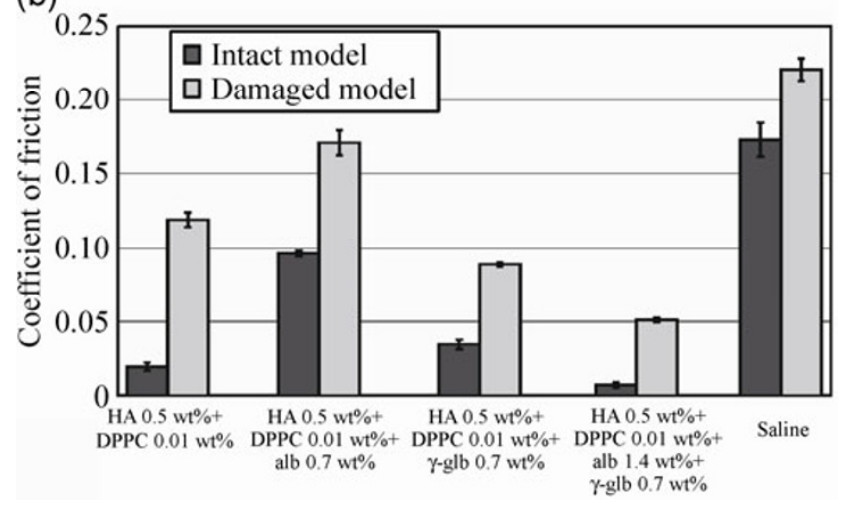

Fig. 9 Friction levels at restart and steady state for intact and damaged cartilage (error bars indicate standard deviation): (a) friction at restart and (b) friction at steady state. articular cartilage has smooth surface with some irregularity (left picture). Tests lubricated with No. 10 lubricant (HA solution containing $0.01 \mathrm{wt} \%$ DPPC) and No. 12 (HA solution containing $0.01 \mathrm{wt} \%$ DPPC and $0.7 \mathrm{wt} \% \gamma$-globulin) exhibited the low friction and mild wear with scratches on surfaces. On the contrary, minimum friction as 0.01 and little wear were confirmed for lubricant No. 13 (HA solution containing $0.01 \mathrm{wt} \%$ DPPC, $1.4 \mathrm{wt} \%$ albumin and 0.7 wt $\%$-globulin), where superficial gel-like layer may have been slightly removed without scratching during rubbing.

\section{Discussion}

For natural synovial joint systems, the synergistic action between articular cartilage and synovial fluid appears to play an important role in minimizing friction and wear. In this study, the repeated reciprocating tests including interrupting-unloading periods for $5 \mathrm{~min}$ for ellipsoidal cartilage specimen against flat glass plate were conducted, where the contact zone of articular cartilage was not migrated and thus the effect of the interstitial fluid pressurization in articular cartilage

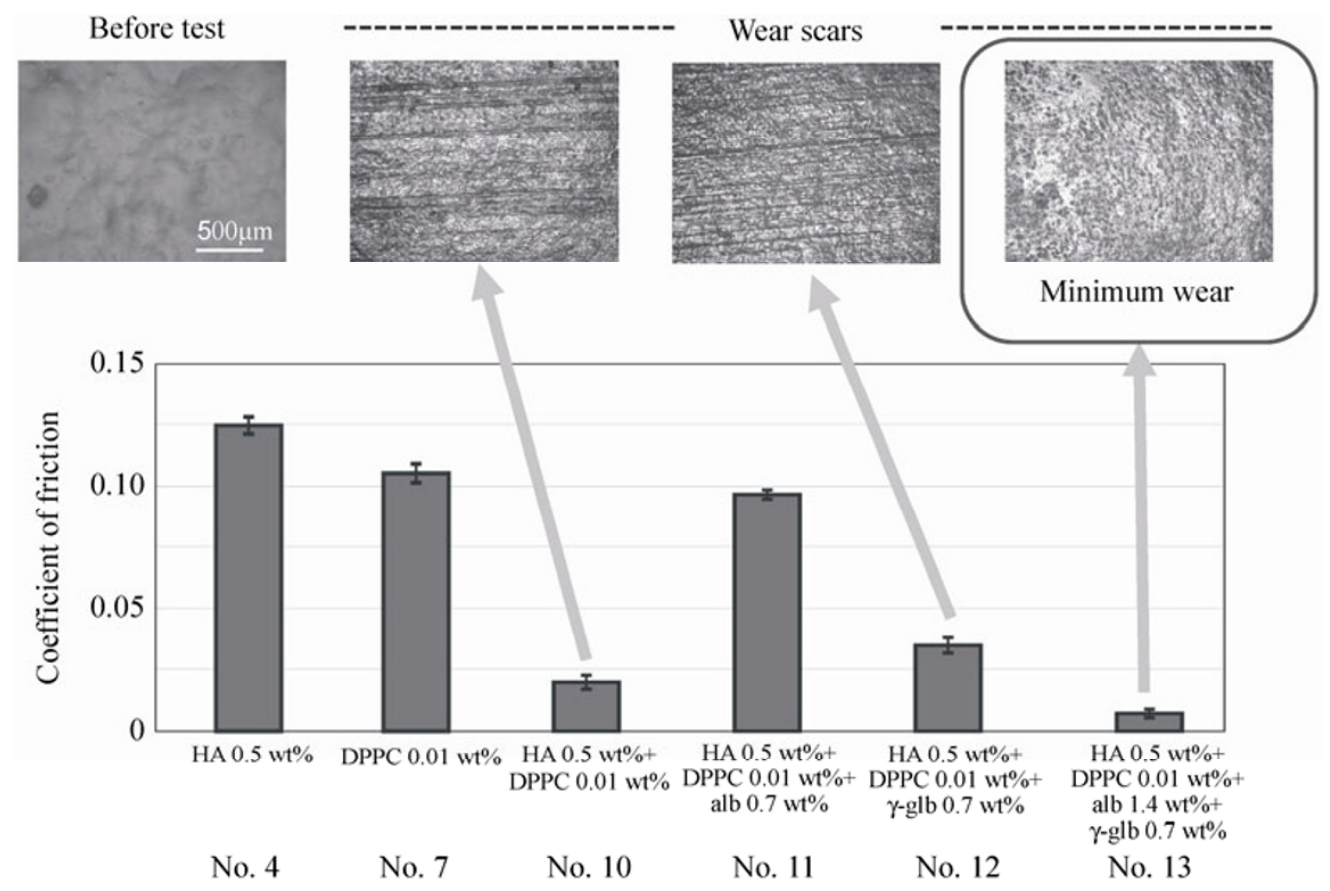

Fig. 10 Average friction levels at steady state and cartilage surface photographs for intact cartilage (error bars indicate standard deviation). 
was gradually diminished. Under such severe rubbing conditions as model B in Fig. 1, the effectiveness of lubricant constituents and the influence of cartilage surface conditions on tribological behaviors were evaluated.

The common features in frictional behaviors of articular cartilage in the reciprocating tests are as follows.

(1) Initial low friction is established by biphasic/ hydration and/or mixed lubrication for cartilage surface with sufficient adsorbed films.

(2) Time-dependent gradual increase in friction during rubbing process is controlled by biphasic property of cartilage, interaction of adsorbed molecules and/or slight removal of cartilage surfaces.

(3) Reduction in restarting friction is brought by the recovery of hydration and biphasic property with recovery of deformation accompanied with adsorbed film formation after unloading for $5 \mathrm{~min}$.

As indicated by the Eq. (1) in FE analysis, we can estimate the frictional behaviors of various cartilage surfaces different in adsorbed film formation, i.e., coefficient of friction for solid-to-solid contact $\mu_{\text {eq }}$. In Fig. 11, the changes in friction estimated from total traction force in biphasic FE analyses during rubbing process under constant load are shown for $\mu_{\text {eq }}=0.01$ and 0.2. Most cases of frictional behaviors in this study except for addition of a single protein seem to be located between the upper (high friction) and lower (low friction) curves in Fig. 11, although FE analysis was conducted for two dimensional model.

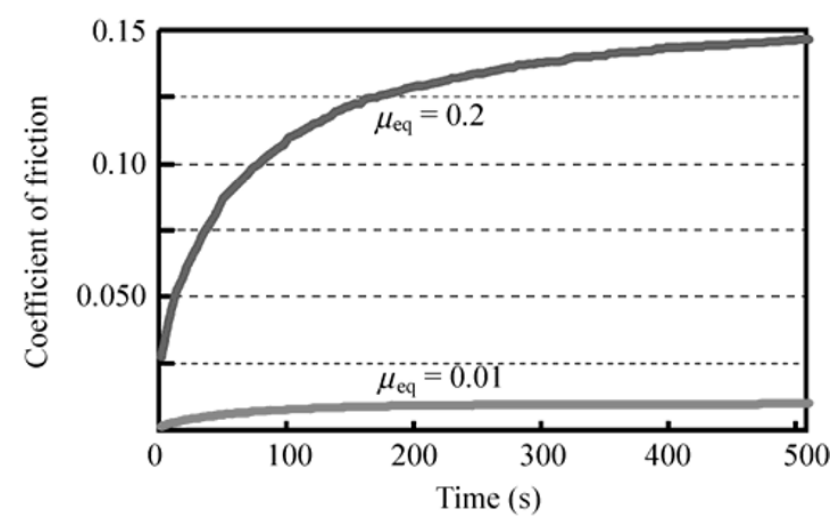

Fig. 11 Influence of $\mu_{\mathrm{eq}}$ on time-depending frictional behaviors estimated by biphasic theory for cartilage.
In cases of addition of a single constituent into saline solution (Fig. 4), the frictional features as described above are observed, but the friction levels change depending on the properties of lubricant constituents. The addition of protein, i.e., albumin or $\gamma$-globulin into saline solution improved the restarting friction but increased the final friction at each $36 \mathrm{~m}$ sliding. Particularly, the addition of $\gamma$-globulin brought a remarkable lowering in restarting friction but higher final friction than albumin. The reason why two kinds of proteins show different friction levels was considered that $\gamma$-globulin has stronger adsorption ability on cartilage than albumin as indicated by fluorescent images [27], and thus $\gamma$-globulin showed lower restarting friction with appropriate adsorbed film formation in mild condition immediately after reloading, but exhibited higher friction due to molecular interaction as a bonding effect in very thin film condition after each $36 \mathrm{~m}$ sliding.

In the in situ observation of the rubbing pair of poly(vinyl-alcohol) (PVA) hydrogel and glass plate by Yarimitsu et al. [28], the fluorescent images for proteins adsorbed on glass plate, protein aggregates between rubbing surfaces and proteins on PVA hydrogel surface were discriminately observed in reciprocating tests for boundary lubrication regime at low sliding speed of $0.2 \mathrm{~mm} / \mathrm{s}$ and the average contact pressure of $0.104 \mathrm{MPa}$. This reciprocating apparatus was constructed on the stage of the inverted fluorescent microscope. In saline solution of albumin, the easy peeling of albumin was observed, but in saline solution of $\gamma$-globulin, quick adsorption and uniform adsorbed film formation were observed. These phenomena indicate the differences in adsorption abilities for both proteins. In binary protein solutions with coexistence of albumin and $\gamma$-globulin, the relative ratio and concentration of proteins had an intense influence on adsorbed film formation [29]. Furthermore, the observation of adsorbed molecules in the evanescent field within about $200 \mathrm{~nm}$ from surface by using the total internal reflection fluorescence (TIRF) microscopy indicated in binary protein solutions that the bottom layer of stable protein adsorbed film is mainly composed of $\gamma$-globulin and the friction-induced enhancement of forming protein adsorbed film occurs 
in lubricant with appropriate protein composition $[30,31]$. The competitive adsorption of albumin and $\gamma$-globulin appears to affect these behaviors as indicated in study of adsorption and desorption of both proteins with TIRF spectroscopy by Tremsina et al. [32]. Furthermore, the differences in adsorption behaviors of serum proteins depend on the changes in conformation, molecular weight, charge condition, hydrophobic/hydrophilic properties of proteins and solid surfaces, $\mathrm{pH}$ of lubricant, and so on. Particularly under rubbing, denatured proteins change their conformations and adsorption properties, and thus affect the tribological behaviors [33-35]. Therefore, overall viewpoints are required to elucidate the actual adsorption behaviors of serum proteins.

The addition of HA with viscous property in lubricants was expected to improve the fluid film thickness, and subsequently improved friction level compared with saline [26]. The addition of DPPC alone is the most effective in reduction of friction but the final coefficient of friction is not so low (about 0.1) in Fig. 4.

Therefore, the effect of combination of different constituents was evaluated. The influences of coexistence of protein with HA on friction were examined in our previous study [26]. The coexistence of $\gamma$-globulin and HA showed the lowering of both the restarting and final or steady friction compared with HA solution. However, albumin exhibited higher final friction than HA solution although it showed a little lower restarting friction than HA solution. These facts suggest the synergistic effect of $\gamma$-globulin and HA, but indicate the adverse interaction of albumin and HA for intact cartilage. It is reported that albumin and HA show repulsive interaction [36] and the HA-protein complexes in natural synovial fluid contain globulin but almost no albumin at $\mathrm{pH}$ 7-8 [37]. These frictional trends for both proteins are similar for damaged cartilage with partially removed surface proteoglycan gel layer. The suppressive action between negatively charged albumin and negatively charged HA molecules was observed in fluorescent images of sparsely distributed adsorbed films, compared with intimate adsorbed films for $\gamma$-globulin and HA [26].

In this study, the effect of addition of neutral phospholipid DPPC with and without protein was examined. It should be noted that the coexistence of DPPC with protein is effective for intact cartilage (Fig. 5), but increases friction for damaged cartilage (Fig. 6). This difference appears to be brought about by changes in adsorbed film formation on damaged cartilage surface. For reciprocating tests of PVA hydrogel and glass plate lubricated with saline solution of DPPC alone, the Janus-faced property for high or low friction was affirmed in accord to either irregular adsorbed film or uniform DPPC adsorbed film formation in AFM images [38]. It is pointed out by Hills [10] that even only the oligolamellar phospholipid plays an effective lubricating role in natural synovial joints. By in situ fluorescent observation of forming adsorbed films for sliding pair of PVA hydrogel and glass plate in coexistence of DPPC and albumin [38], it was clarified that the formation of albumin-DPPC sheet-like composite film was found and therefore the friction was reduced. It is pointed out that DPPC with a neutral charge is likely to bind to albumin [39].

Next, the influence of addition of DPPC in HA solution with and without proteins was examined. The addition of DPPC alone in HA solution was considerably effective in reduction of friction for intact cartilage compared with coexistence of DPPC and either albumin or $\gamma$-globulin in HA solution (Fig. 7). This fact may suggest the formation of lubricating complex materials as membrane-like and roller structures composed of DPPC and HA [40]. Mirea et al. [41] indicated that $\mathrm{HA}$ has high affinity to phospholipid bilayer in the force-distance curve in AFM study. The detailed structure of HA-DPPC complex has not yet been clarified but the coexistence of DPPC and HA is likely to act synergistically as lamellar lubrication or related mechanism. Furthermore, for coexistence of DPPC and HA, HA-DPPC composite boundary film was visually confirmed [38] and friction was remarkably lowered, where the lubricating ability by HA-DPPC complex as gel-like film is supposed to become effective with high water retention ability of HA. However, HA solution containing DPPC showed an effective but limited protective property with local scratching as shown in Fig. 10.

On the contrary, albumin-DPPC composite was not 
found in coexistence of three constituents, i.e., DPPC, HA and albumin [38], probably due to repulsive interaction between albumin and HA. This fact corresponds to the phenomenon in which the friction for HA solution with DPPC and albumin (Fig. 7) is higher than saline solution with DPPC and albumin (Fig. 5).

However, the supply of both albumin and $\gamma$-globulin as definite ratio into HA solution containing DPPC (lubricant No.13) could remarkably improve the friction at very low level of 0.01 as final coefficient of friction (Fig. 7) and high wear resistance (Fig. 10). For damaged cartilage, the friction level increased in general but No.13 lubricant showed the minimum friction (Fig. 8).

In natural synovial joints, various lubricating constituents such as HA, proteins, glycoproteins and phospholipds different in molecular properties and sizes play different roles. Therefore, the interaction and/or synergistic action between phospholipids and other constituents seem to control the adsorbed film formation and tribological behavior. The influences of lubricants as HA solutions containing DPPC with or without proteins on the friction at restart and at steady state are summarized in Fig. 9 for intact and damaged cartilage specimens. The effectiveness of adsorbed film on reduction in restarting friction and steady friction is clearly demonstrated compared with saline solution. Particularly, it is noticed that the lubricant No.13 (HA solution with $1.4 \mathrm{wt} \%$ albumin, $0.7 \mathrm{wt} \%$-globulin and $0.01 \mathrm{wt} \%$ DPPC) provided very low restarting friction for both intact and damaged cartilage specimens (Fig. 9(a)). This lubricant maintained very low friction until each $36 \mathrm{~m}$ sliding for intact cartilage, but the friction gradually increased until 0.05 as coefficient of friction for damaged cartilage (Fig. 9(b)). In the study by Nakashima et al. [29], HA solution with $1.4 \mathrm{wt} \%$ albumin and $0.7 \mathrm{wt} \%$ $\gamma$-globulin (albumin/globulin $=\mathrm{A} / \mathrm{G}$ ratio of $2: 1$ ) or $0.7 \mathrm{wt} \%$ albumin and $1.4 \mathrm{wt} \% \gamma$-globulin (A/G ratio of 1:2) showed very low wear for rubbing of PVA hydrogel against itself. For low wear condition in the latter, the layered adsorbed film formation was observed by the fluorescent method. In these cases, it is suggested that the $\gamma$-globulin forms protective adsorbed layer on cartilage surface and albumin plays as low shearing layers. On the contrary, HA solution with $1.4 \mathrm{wt} \%$ albumin and $1.4 \mathrm{wt} \% \gamma$-globulin $(\mathrm{A} / \mathrm{G}$ ratio of 1:1) formed the heterogeneous adsorbed film and showed higher wear.

The lubricant No.13 has similar composition to that in natural synovial fluid as hyaluronate solution containing lubricating constituents such as $1.25 \mathrm{wt} \%$ albumin, $0.75 \mathrm{wt} \%$ globulin (including $\alpha-, \beta-$, and $\gamma$-globulins) as medium values [42], $1.1 \mathrm{wt} \%$ albumin and $0.7 \mathrm{wt} \%$ globulin [36], or $1.9 \mathrm{wt} \%$ albumin, $1.1 \mathrm{wt} \%$ globulin and $0.01 \mathrm{wt} \%$ DPPC [12]. In this lubricant, the lubricating layered structure in adsorbed film is expected for low friction and minimal wear, but the detailed elucidation of this mechanism is required in the future study. As exhibited in Figs. 7 and 9, lubricant No. 13 showed very low and steady friction in repeated reciprocating test at $20 \mathrm{~mm} / \mathrm{s}$. In situ fluorescent observation at very slow speed with this lubricant [38] showed the stable mixed adsorbed film containing albumin and $\gamma$-globulin but friction is not so low probably due to very thin film condition at $0.2 \mathrm{~mm} / \mathrm{s}$ condition. Therefore, we plan to observe in situ the actual adsorbed film formation and frictional behavior at $20 \mathrm{~mm} / \mathrm{s}$ or so. In various daily activities, synovia constituents appear to play their appropriate roles depending on the severity of operating conditions. DPPC and albumin are likely to act as low shearing layer, and $\gamma$-globulin acts as the protective film as strongly adsorbed on cartilage surface. HA has ability to thicken the lubricating fluid film and form some lubricating gel-like layer. Although some of synergistic mechanisms between lubricating constituents were shown in this study, the overall mechanisms are expected to be clarified from the viewpoint of multiscale level in future. On the role of lubricin as another lubricating constituent, Mirea et al. [41] suggested that it anchors lipid layers on the cartilage. We confirmed that the addition of lubricin in HA solution could reduce friction for intact cartilage in the preliminary test. In future study, we plan to evaluate the effective roles of all influential synovia constituents.

For damaged cartilage specimens with partially removed proteoglycan brush-like layer, the best composition in lubricant for low friction is the same lubricant No. 13 which is the best for intact cartilage, but the second one was changed to the HA solution 
containing DPPC with $\gamma$-globulin from the HA containing DPPC solution without protein as the second one for intact cartilage. It is suggested for damaged cartilage that the protective role of $\gamma$-globulin with strong adsorption ability becomes important.

As discussed above, the effectiveness of lubricant constituents changes depending on rubbing cartilage properties in reciprocating tests of cartilage-on-glass. To evaluate rigorously the influence of synovia constituents on tribological behaviors of articular cartilage in natural synovial joints, the rubbing pair of cartilageon-cartilage [8,43] or cartilage-on-meniscus [44] should be used, and therefore the influence of glass plate on tribological behaviors in this study should be discussed. As mentioned in Section 1, the glass plate surface possesses hydrophilic characteristics with negatively charged property similar to proteoglycan on superficial cartilage layer in wet condition, whilst it is hard, smooth and nonporous/impermeable material. The adsorption of synovia constituents on glass plate is expected to be considerably similar to boundary film formation on intact cartilage but the interaction to the smooth, hard and nonporous/ impermeable glass surface may be different. HA and albumin (at $\mathrm{pH}>4.7$ ) are negatively charged but $\gamma$-globulin is positively charged (at $\mathrm{pH}<7.5$ ). These electrostatic properties of adsorbed molecules have an influence on adsorption. On the contrary, the ploughing friction may be minimized for smooth surface, but adsorbed proteins on very smooth surface may induce high friction by their intense adhesive effect as hydrophobic bonding in watery system in very thin film condition. However, the effectiveness of lubricant constituents on tribological behaviors of compliant and biphasic articular cartilage appear to be reflected appropriately even in sliding pair of articular cartilage and glass plate. In pendulum friction tests for cartilage-on-cartilage of porcine shoulder joints composed of humerus head and glenoid cavity (cup) [8], the effectiveness in friction reduction by addition of $0.01 \mathrm{wt} \%$ DPPC or $1.0 \mathrm{wt} \% \gamma$-globulin to HA solution for cartilage treated with detergent had been confirmed as similar effect to cartilage-on-glass combination. In contrast, the addition of $1.0 \mathrm{wt} \%$ or $3.0 \mathrm{wt} \%$ albumin to HA solution did not improve friction of cartilage-on-cartilage, which corresponds to adverse interaction of albumin and HA for cartilageon-glass [26]. In contrast, the sliding pair of cartilage and clean glass plate showed higher friction in HA solution than that of cartilage and glass plate treated with Langmuir-Blodgett (LB) film as 5 to 10 bilayer of DPPC alone or mixed LB film of DPPC and $\gamma$-globulin [12]. As mentioned above, common features and/or some differences seem to occur in frictional behavior for cartilage-glass combination compared with cartilage-cartilage. In the next stage, therefore, further studies for cartilage-on-cartilage or cartilage-onhydrogel (artificial cartilage) are required to elucidate strictly the influence of synovia constituents on tribological behaviors of articular cartilage in natural synovial joints. The sustaining of the synergistic mechanism of various synovia constituents on matched cartilage surfaces in natural synovial joints is expected to maintain the healthy condition.

\section{Conclusions}

In this study, at repeated reciprocating tests including restarting after interrupting-unloading process, the changes in friction were observed for intact and damaged articular cartilage specimens against glass plate lubricated with lubricants containing phospholipid, protein and hyaluronic acid as synovia constituents. The optimum composition in lubricants for low friction and minimum wear of both intact and damaged cartilage specimens was exhibited to be similar composition to natural synovial fluid. Furthermore, it was shown that the effectiveness of lubricant constituents changes depending on the surface conditions of articular cartilage.

\section{Acknowledgements}

Financial support was given by the Grant-in-Aid for Specially Promoted Research of Japan Society for the Promotion of Science (23000011).

Open Access: This article is distributed under the terms of the Creative Commons Attribution Noncommercial License which permits any noncommercial use, distribution, and reproduction in any medium, provided the original author(s) and source are credited. 


\section{References}

[1] Dowson D. Modes of lubrication in human joints. Proc Inst Mech Engrs 181: 45-54 (1966)

[2] Unsworth A, Dowson D, Wright V. Some new evidence on human joint lubrication. Ann Rheum Dis 34(4): 277-285 (1975)

[3] McCutchen CW. The frictional properties of animal joints. Wear 5: 1-17 (1962)

[4] Walker P S, Dowson D, Longfield M D, Wright V. Boosted lubrication in synovial joints by fluid entrapment and enrichment. Ann Rheum Dis 27(6): 512-520 (1968)

[5] Mansour J M, Mow V C. On the natural lubrication of synovial joints: Normal and degenerate. J Tribol 99: 163-173 (1977)

[6] Dowson D, Jin Z M. Micro-elastohydrodynamic lubrication of synovial joints. Eng Med 15: 65-67 (1986)

[7] Murakami T. The lubrication in natural synovial joints and joint prostheses. JSME International Journal Ser III 33(4): 465-474 (1990)

[8] Murakami T, Higaki H, Sawae Y, Ohtsuki N, Moriyama S, Nakanishi Y. Adaptive multimode lubricaion in natural synovial joints and artificial joints. Proc Inst Mech Eng Part H 212: 23-35 (1998)

[9] Swann D A, Hendren R B, Radin E L, Sotman S L, Duda E A. The lubricating activity of synovial fluid glycoproteins. Arthritis Rheum-US 24: 22-30 (1981)

[10] Hills B A. Oligolamellar lubrication of joints by surface active phospholipids. $J$ Rheum 16(1): 82-91 (1989)

[11] Higaki H, Murakami T. Role of constituents in synovial fluid and surface layer of articular cartilage in joint lubrication (part 2) the boundary lubricating ability of proteins. Jpn J Tribol 40(7): 691-699 (1996)

[12] Higaki H, Murakami T, Nakanishi Y, Miura H, Mawatari T, Iwamoto $\mathrm{Y}$. The Lubricating ability of biomembrane models with dipalmitoyle phosphatidylcholine and $\gamma$-globulin. Proc Inst Mech Eng Part H 212: 337-346. (1998)

[13] Murakami T, Sawae Y, Horimoto M, Noda M. Role of surface layers of natural and artificial cartilage in thin film lubrication. In Lubrication at Frontier. A msterdam: Elsevier, 1999: 737-747.

[14] Ikeuchi K. Origin and future of hydration lubrication. Proc Inst Mech Eng Part J 221: 301-305 (2007)

[15] Klein J. Molecular mechanisms of synovial joint lubrication. Proc Inst Mech Eng Part J 220: 691-710 (2006)

[16] Zappone B, Ruths M, Greene W G, Jay G D, Israelachvili J N. Adsorption, lubrication, and wear of lubricin on model surfaces: Polymer brush-like behavior of a glycoprotein. Biophysical J 92: 1693-1708 (2007)
[17] Forster H, Fisher J. The influence of loading time and lubricant on the friction of articular cartilage. Proc Inst Mech Eng Part H 210: 109-119 (1996)

[18] Ateshian G A. Theoretical formulation for boundary friction in articular cartilage. J Biomech Eng 119(1): 81-86 (1997)

[19] Krishnan R, Kopacz M, Ateshian G A. Experimental verification of the role of interstitial fluid pressurization in cartilage lubrication. J Orthop Res 22: 565-570 (2004)

[20] Ateshian G A. The role of interstitial fluid pressurization in articular cartilage lubrication. J Biomech 42: 1163-1176 (2009)

[21] Mow V C, Kuei S C, Lai W M, Armstrong C G. Biphasic creep and stress relaxaion of articular cartilage in compression: Theory and experiments. ASME J Biomech Eng 102: 73-84 (1980)

[22] Pawaskar S S, Jin Z M, Fisher J. Modelling of fluid support inside articular cartilage during sliding. Proc Inst Mech Eng Part J 221: 165-174 (2007)

[23] Sakai N, Hagihara Y, Furusawa T, Hosoda N, Sawae Y, Murakami T. Analysis of biphasic lubrication of articular cartilage loaded by cylindrical indenter. Tribol Int $\mathbf{4 5}$ : 225-236 (2012)

[24] Murakami T. Importance of adaptive multimode lubrication mechanism in natural and artificial Joints. Proc Inst Mech Eng Part J 216: 827-837 (2012)

[25] Ateshian G A, Wang H, Lai W M. The role of interstitial fluid pressurization and surface porosities on the boundary friction of articular cartilage. ASME J Tribol 120: 241-248 (1998)

[26] Murakami T, Nakashima K, Yarimitsu S, Sawae Y, Sakai N. Effectiveness of adsorbed film and gel layer in hydration lubrication as adaptive multimode lubrication mechanism for articular cartilage. Proc Instn Mech Eng Part J 225: 1174-1185 (2011)

[27] Murakami T, Nakashima K, Sawae Y, Sakai N, Hosoda N. Roles of adsorbed film and gel layer in hydration lubrication for articular cartilage. Proc Inst Mech Eng Part J 223: 287-295 (2009)

[28] Yarimitsu S, Nakashima K, Sawae Y, Murakami T. Study on mechanisms of wear reduction of artificial cartilage through in situ observation on forming protein boundary film. Tribol Online 2(4): 114-119 (2007)

[29] Nakashima K, Sawae Y, Murakami T. Study on wear reduction mechanisms of artificial cartilage by synergistic protein boundary film formation. JSME Int J 48(4): 555-561 (2005)

[30] Yarimitsu S, Nakashima K, Sawae Y, Murakami T. Effect of lubricant composition on adsorption behavior of proteins on rubbing surface and stability of protein boundary film. Tribol Online 3(4): 238-242 (2008) 
[31] Yarimitsu S, Nakashima K, Sawae Y, Murakami T. Influences of lubricant composition on forming boundary film composed of synovia constituents. Tribol Int 42: 1615-1623 (2009)

[32] Tremsina Y S, Sevastianov V I, Petrash S, Dando W, Foster M D. Competitive adsorption of human serum albumin and gamma-globulin from a binary protein mixture onto hexadecyltrichlorosilane coated glass. J Biomater Sci Polym Ed 9(2): 151-162 (1998)

[33] Heuberger M P, Widmer M R, Zobeley E, Glockshuber R, Spencer N D. Protein-mediated boundary lubrication in arthroplasty. Biometarials 26: 1165-1173 (2005)

[34] Nakashima K, Sawae Y, Murakami T. Influence of protein conformation on frictional properties of poly)vinyl alcohol) hydrogel for artificial cartilage. Tribol Lett 26: 145-151 (2007)

[35] Nakashima K, Sawae Y, Murakami T. Effect of conformational changes and differences of proteins on frictional properties of poly(vinyl alcohol) hydrogel. Tribol Int 40: 1423-1427 (2007)

[36] Oates K M N, Krause W E, Jones R L, Colby R H. Rheopexy of synovial fluid and protein aggregation. $J R$ Soc Interface 3: 167-174 (2006)

[37] Curtain C C. The nature of protein in the hyaluronic complex

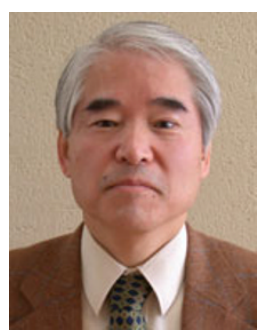

Teruo MURAKAMI. Professor at Research Center for Advanced Biomechanics, Kyushu University. He graduated from Kyushu University in 1970 and received his PhD degree from Kyushu University in 1978. He was appointed a professor of of bovine synovial fluid. Biochem J 61(4): 688-697 (1955)

[38] Yarimitsu S, Nakashima K, Sawae Y, Murakami T. Influences of synovia constituents on frictional behavior of artificial cartilage material and formation of boundary lubricating film (in Japanese). Tribologist 55(7): 489-498 (2010)

[39] Hernández-Caselles T, Villalaín J, Gómez-Fernáindez J C. Influence of liposome charge and composition on their interaction with human blood serum proteins. Mol Cell Biochem 120: 119-126 (1993)

[40] Pasquali-Ronchetti I, Quaglino D, Mori G, Bacchell B. Hyaluronan-phospholipid interactions. J Struct Biol 120: 1-10 (1997)

[41] Mirea D A, Trunfio-Sfarghiu A-M, Matei C I, Munteanu B, Piednoir A, Rieu J P, Blanchin M G, Berthie Y. Role of the biomolecular interactions in the structure and tribological properties of synovial fluid. Tribol Int 59: 302-311 (2013)

[42] Sasada T, Tsukamoto Y, Mabuchi K. Biotribology (in Japanese). Sangyo Tosho, 1988.

[43] Roberts B J, Unsworth A, Mian N. Modes of lubrication in human hip joints. Ann Rheum Dis 41: 217-224 (1982)

[44] McCann L, Ingham E, Jin Z, Fisher J. Influence of the meniscus on friction and degradation of cartilage in natural knee joint. Osteoarthr Cartilage 17: 995-1000 (2009)

Mechanical Engineering in 1988 and a distinguished professor in 2011 at Kyushu University. Research fields are biotribology, biomechanics and bionic design. $\mathrm{He}$ is a research leader of a Grant-in-Aid for Scientific Research on artificial hydrogel cartilage with super lubricity as Specially Promoted Research supported by Japan Society for the Promotion of Science. 